\title{
Lidil
}

Revue de linguistique et de didactique des langues

Multimodalité de la communication chez l'enfant

\section{Interactions mère-enfant typique et dysphasique : comment utiliser les gestes pour formuler une devinette?}

Geneviève de Weck, Anne Salazar Orvig, Cristina Corlateanu, Christine da Silva, Stefano Rezzonico et Tiziana Bignasca

\section{OpenEdition}

Édition électronique

URL : http://journals.openedition.org/lidil/3084

DOI : 10.4000/lidil.3084

ISSN : 1960-6052

Éditeur

UGA Éditions/Université Grenoble Alpes

\section{Édition imprimée}

Date de publication : 30 novembre 2010

Pagination : 159-180

ISBN : 978-2-84310-184-7

ISSN : $1146-6480$

Référence électronique

Geneviève de Weck, Anne Salazar Orvig, Cristina Corlateanu, Christine da Silva, Stefano Rezzonico et Tiziana Bignasca, «Interactions mère-enfant typique et dysphasique : comment utiliser les gestes pour formuler une devinette ? », Lidil [En ligne], 42 | 2010, mis en ligne le 31 mai 2012, consulté le 03 mai 2019. URL : http://journals.openedition.org/lidil/3084 ; DOI : 10.4000/lidil.3084 


\title{
Interactions mère-enfant typique et dysphasique : comment utiliser les gestes pour formuler une devinette?
}

\author{
Geneviève de Weck*, Anne Salazar Orvig**, \\ Cristina Corlateanu* et**, Christine da Silva* et**, \\ Stefano Rezzonico* *t** et Tiziana Bignasca*
}

RÉSUMÉ

Cet article étudie l'usage des gestes par 18 dyades mère-enfant dysphasique en comparaison avec 18 dyades mère-enfant typique dans une situation de jeu de devinette par indices. Tous les enfants sont âgés entre 5 et 7 ans.

Les analyses concernent la proportion, les rôles ainsi que la nature sémiotique des gestes utilisés comme indices dans le jeu de devinettes. Les résultats aboutissent à des différences quantitatives et qualitatives dans l'emploi des gestes entre les deux types de dyades. Même si globalement la devinette se profile comme une activité essentiellement verbale, il apparait que les enfants mobilisent plus souvent les gestes pour la production d'indices que leurs mères et que les dyades mère-enfant dysphasique présentent des taux plus élevés de gestes que les dyades mère-enfant typique.

Par ailleurs, les résultats mettent en évidence des spécificités chez les enfants dysphasiques. Ils semblent utiliser davantage de gestes déictiques et certains d'entre eux ont recours plus souvent aux gestes illustratifs, se substituant au verbal. L'étude montre également une certaine hétérogénéité des dyades dans les deux groupes de la population.

\section{ABSTRACT}

This paper investigates the use of gestures by 18 mother-SLI children dyads compared to 18 mother-typically developing children dyads in a guessing game situation. All children are between 5 and 7 years old.

* Université de Neuchâtel.

** Université Sorbonne Nouvelle-Paris 3. 
Analyses concern the proportion, the roles and the semiotic nature of gestures used as clues in the guessing game. Results show both quantitative and qualitative differences between the two types of dyads. Even though the guessing game stands out as an essentially verbal activity, it appears that children make use of gestures as clues more frequently than their mothers and that mother-SLI children dyads produce a higher rate of gestures than mother-TD children dyads.

In addition, results highlight several specific characteristics of SLI children. They seem to produce more deictic gestures and some of them make use of more illustrative gestures as substitutes for verbal productions. The study also shows that, to some extent, dyads in both types of population are quite heterogeneous (in their behavior).

Cet article présente les premiers résultats d'une étude sur la place des gestes dans les interactions mère-enfant dysphasique. Cette étude ${ }^{1} \mathrm{~s}$ 'inscrit dans une recherche plus large sur les modes de gestion des dialogues par les mères et leurs enfants dans diverses activités langagières. Nous nous focalisons ici plus spécifiquement sur l'usage des gestes par des enfants de 5 à 7 ans et leurs mères lors d'une activité de devinettes. Cette étude vise à mieux comprendre cet usage et ses liens avec les troubles du développement du langage présentés par une partie de la population étudiée.

Les troubles dysphasiques² (Rapin et Allen, 1983; Gérard, 1991 ; de Weck et Rosat, 2003 par exemple) se manifestent très souvent par des troubles aux niveaux phonologique et syntaxique (syndrome phono-

1. Cette étude s'inscrit dans un programme de recherche intitulé «Interactions mère-enfant en situation logopédique» financé par le FNRS suisse (subside $\mathrm{n}^{\circ}$ 100012-111938) et mené par les deux équipes, à Neuchâtel et à Paris 3 , dirigées respectivement par G. de Weck et A. Salazar Orvig; il a impliqué, en plus des auteurs, d'autres collaboratrices (Séverine Gendre, Juliane Ingold et Céline Schwab Stoebener). Qu'elles soient ici remerciées ainsi que les mères, les enfants et les logopédistes-orthophonistes, en Suisse romande et en France, qui nous ont mis en contact avec les familles des enfants dysphasiques.

2. Ces troubles sont également appelés «troubles spécifiques du langage» et plus précisément «troubles spécifiques du développement du langage» (cf. par exemple Chevrie-Muller et Narbonna, 2000) qui est proche du terme anglophone «Specific Language Impairment». Cependant, dans le contexte francophone, le terme le plus fréquemment utilisé est celui de «dysphasie». Dans un cas comme dans l'autre, il s'agit d'un syndrome défini par exclusion d'autres types de troubles (neurologiques, sensoriels, psychiques...). 
logique-syntaxique). Ces troubles de la structure de la langue se distinguent des troubles pragmatiques (syndrome sémantique-pragmatique; Bishop, 2000; Rapin et Allen, 1983) qui concernent la gestion du dialogue et de l'interaction. Ainsi, en principe, on n'observe pas de troubles pragmatiques chez les enfants présentant une dysphasie de type phonologique-syntaxique. Or, plusieurs études commencent à montrer que les choses ne sont pas aussi claires. Même si les troubles de la structure linguistique dominent chez les dysphasiques, ils ont des retentissements sur les modes d'interaction, de sorte que des troubles pragmatiques secondaires peuvent apparaitre. Par ailleurs, jusqu'ici l'intérêt des chercheurs s'est beaucoup focalisé sur les aspects langagiers, et relativement peu de données sont disponibles sur les aspects non verbaux de la communication, et en particulier sur l'emploi des gestes par les enfants et leurs mères.

Parmi les difficultés pragmatiques que rencontrent les enfants dysphasiques, leur passivité dans l'interaction est souvent mentionnée (Conti-Ramsden, 1994; Conti-Ramsden et al., 1995; Hupet, 1996), les adultes prenant la plupart du temps les initiatives. En conséquence, les enfants ont plutôt tendance à réduire leurs prises de parole à des interventions minimales, souvent en réponse aux interventions de leur interlocuteur. Par ailleurs, leurs prises de parole ne sont pas toujours appropriées (Bishop et al., 2000). Des difficultés à se représenter les besoins de leur interlocuteur (de Weck et Rosat, 2003) ont également été relevées.

Toutefois, ces difficultés pragmatiques ont été observées en tenant compte uniquement de la dimension verbale. La question qui se pose alors est celle de l'usage des gestes par les enfants dysphasiques. On pourrait faire l'hypothèse qu'ils les utilisent davantage que des enfants typiques, soit à la place de leurs prises de parole soit en complémentarité avec celles-ci, afin de tenter de compenser un peu leurs difficultés verbales (Bishop et al., 1998). Il est également intéressant de s'interroger sur la nature des gestes auxquels ils recourent. De même, les mères utilisent-elles les gestes de façon analogue, indépendamment de la présence ou non de troubles chez leur enfant?

Les données relatives à l'emploi des gestes par les enfants dysphasiques sont encore rares et controversées. Pour Botting et collaborateurs (Botting et al., 2010), les enfants dysphasiques ne se distinguent pas des enfants typiques sur le plan de la production des gestes. De même, dans une étude comparant des enfants dysphasiques âgés entre 5 et 10 ans avec des enfants de même âge chronologique et mental, d'une part, 
et des enfants de même niveau langagier, Blake et ses collaborateurs (Blake et al., 2008) ont montré que même si les enfants dysphasiques produisent davantage de gestes iconiques que les enfants contrôle, le niveau langagier n'a pas un effet clair sur le système gestuel. Au contraire, d'après d'autres études, (Capone et McGregor, 2004, pour une synthèse), les gestes chez les enfants dysphasiques semblent être un moyen de communication semblable à celui des jeunes enfants, avec des moyens linguistiques encore insuffisamment développés, confirmant l'étroite relation développementale entre les modalités gestuelle et verbale, attestée chez les enfants dès la période prélinguistique. Plus précisément, des études longitudinales (Thal et Tobias, 1992) se sont intéressées à l'emploi des gestes chez les enfants présentant un retard dans l'apparition du langage. Elles ont montré une différence entre les enfants qui récupèrent leur retard vers 3 ans et ceux dont le retard persiste au-delà de cet âge. Les premiers semblent avoir utilisé davantage de gestes, à la fois déictiques et symboliques, dans les premières années pour compenser leur retard verbal et exprimer des intentions communicatives; ils ont même utilisé davantage de gestes déictiques que les enfants typiques de même niveau linguistique. Par contre, les enfants avec un retard de langage persistant manifestent également un déficit dans l'utilisation des gestes. Mais ceci ne semble pas être toujours le cas. En effet, Mermoud et de Weck (1992) ont décrit l'usage des gestes par une enfant de 4;6 ans présentant un important retard de langage. Parlant très peu, cette enfant a pourtant construit un système de communication organisé dans lequel le non-verbal tient une place centrale : elle utilise de nombreux gestes syllinguistiques et quasilinguistiques (Cosnier, 1977; Dahan et Cosnier, 1977) dans différentes fonctions (illustrative, référentielle, notamment) et de différentes formes (arbitraire, déictique, iconique, notamment). Cette utilisation varie en fonction des activités langagières proposées (jeu symbolique, conversation, lecture d'un livre). Ces résultats vont dans le sens des tendances décrites par Bishop et al. (2000), à savoir des enfants dysphasiques qui utilisent les gestes et d'autres qui n'y ont pas recours. Dans le dernier cas, on peut se demander, avec ces auteurs, si l'absence de recours aux ressources gestuelles ne manifeste pas une difficulté communicationnelle plus grave.

Par ailleurs, l'emploi des gestes semble permettre aux enfants dysphasiques une meilleure production de messages. Evans et al. (2001) ont constaté que des enfants dysphasiques de 7-9 ans, dans une situation d'explication d'une tâche piagétienne de conservation, utilisent les 
gestes dans des proportions semblables à celles des enfants de même niveau cognitif. Toutefois, ils semblent exprimer par des gestes des informations non contenues dans leurs verbalisations d'une part et des connaissances plus élaborées lorsque les gestes et la parole se combinent d'autre part. Ces observations ne se retrouvent pas chez les enfants typiques de même niveau cognitif. Plus spécifiquement, dans le contexte de narrations et de descriptions (Blake et al., 2008), il apparait que les gestes iconiques, plus fréquents chez les enfants dysphasiques, précèdent ou co-occurrent avec le concept mis en mot, ce qui pourrait suggérer qu'ils facilitent l'accès lexical, par amorçage des traits sémantiques.

Enfin, les gestes semblent jouer un rôle dans l'acquisition du vocabulaire. Selon Ellis Weismer et Hesketh (1993), le fait d'accompagner avec des gestes la présentation de nouveaux mots à des enfants dysphasiques et typiques de 5-6 ans a pour effet une meilleure compréhension des mots cibles par les deux types d'enfants que quand la présentation de nouveaux mots est effectuée sans gestes; mais l'effet est plus important chez les enfants dysphasiques. D'une façon plus générale, les gestes semblent avoir pour les enfants dysphasiques (Botting et al., 2010) un véritable effet d'étayage quand il s'agit de comprendre des énoncés.

Alors qu'un certain nombre d'études ont mis en évidence l'existence d'un étayage gestuel ( «gestural motherese») pour le jeune enfant tout venant (Iverson et al., 1999), peu d'études portent sur les développements atypiques. Par exemple, les mères d'enfants souffrant du syndrome de Down adaptent (Iverson et al., 2006) leur gestualité au niveau de développement de l'enfant, utilisent plus de déictiques et montrent davantage qu'elles ne pointent. Surtout elles maintiennent leurs gestes tout au long de l'émission de l'énoncé. Comme pour les mères d'enfants plus jeunes, ces gestes semblent donc avoir comme rôle de renforcer le message verbal. Bien qu'il n'y ait quasi pas de travaux sur l'usage des gestes par les interlocuteurs des enfants dysphasiques (voir par exemple Panagos et al. [1988], analysant les aspects non verbaux des cliniciens s'adressant à des enfants dysphasiques), et notamment des mères, on pourrait extrapoler, à partir des tendances présentées, un effet bénéfique de l'emploi des gestes par les mères sur le fonctionnement interactif : les gestes pourraient permettre aux enfants dysphasiques de mieux comprendre les interventions de leur interlocuteur.

Dans une étude précédente sur les interactions dans une situation de devinette (Salazar Orvig et de Weck, 2008), nous avons montré que, 
malgré leurs difficultés linguistiques avérées, les enfants dysphasiques mobilisent, dans des proportions similaires à celles des enfants typiques, les mêmes types d'interventions : ils ont autant recours à des tours de parole non verbaux ou mixtes (comprenant des éléments verbaux et non verbaux) que les enfants typiques. Cependant, si ces derniers produisent une grande majorité de gestes indépendants du verbal et correspondant le plus souvent aux mouvements du jeu en cours, les enfants dysphasiques semblent mobiliser davantage le non-verbal à la place de l'activité verbale.

Quant aux mères, elles réalisent plus de tours de parole purement verbaux que leurs enfants. Cependant, comme leurs enfants, les mères des enfants dysphasiques mobilisent le non verbal bien plus dans la situation de devinette que dans d'autres situations de communication (de Weck et Salazar Orvig, 2010). Mais contrairement à leurs enfants, le non-verbal vient chez elles surtout redoubler le verbal.

Prenant comme point de départ ces résultats, nous nous focalisons ici sur l'activité de devinette. On peut, en effet, se demander si les enfants dysphasiques mobilisent des gestes pour produire des indices qui ne peuvent être formulés verbalement, et dans l'affirmative, au moyen de quels types de gestes. Corrélativement, les mêmes questions s'appliquent aux mères : les mères des enfants dysphasiques ayant tendance à mobiliser des gestes de façon redondante à leur discours dans l'ensemble de l'activité, est-ce le cas également pour les énoncés de devinette? Quel rôle jouent alors les gestes dans cette situation?

\section{Méthodologie}

Nous présentons ci-dessous la population étudiée, la situation d'interaction qui leur a été proposée ainsi que les axes d'analyses retenus afin de rendre compte de la place des gestes mobilisés dans les séquences de devinettes des dyades mère-enfant.

\section{Population}

La population est constituée de 36 dyades mère-enfant. Les enfants se répartissent en deux groupes nosographiques, des enfants dysphasiques et des enfants typiques de 5 à 7 ans. Les observations ont été effectuées en Suisse romande et en France, dans des centres où travaillent des logopédistes-orthophonistes pour les enfants dysphasiques et dans des écoles maternelles et primaires pour les enfants typiques. La population se distribue de la façon suivante : 
- 18 enfants présentant une dysphasie de type phonologiquesyntaxique (D par la suite) : 12 garçons et 6 filles;

- 18 enfants typiques (T par la suite) : 12 garçons et 6 filles.

Les enfants dysphasiques ont été appariés un par un selon l'âge (un mois de différence au maximum), le sexe et la région avec les enfants typiques. La suprématie des garçons reflète une situation générale bien connue, à savoir que les garçons présentent plus fréquemment que les filles des troubles dysphasiques. Le diagnostic de dysphasie phonologique-syntaxique (Gérard, 1991) ${ }^{3}$ a été posé par les professionnels en charge de ces enfants qui bénéficient par ailleurs d'un traitement logopédique-orthophonique.

Tous les enfants participant à cette recherche ont été soumis à la passation de 6 épreuves évaluant leurs compétences aux niveaux de la phonologie et de la syntaxe - en compréhension et en production tirées des «Nouvelles épreuves pour l'examen du langage» (N-EEL, Chevrie-Muller et Plaza, 2001). Les résultats permettent de confirmer les écarts entre les deux groupes d'enfants, les enfants D présentant au moins 2 écarts-types en dessous de la moyenne de leur âge; les enfants $\mathrm{T}$ se situent à la moyenne de leur âge ou au-dessus. Plus précisément, en se référant à l'âge verbal défini à partir du test, les enfants dysphasiques se répartissent comme suit : 10 enfants obtiennent un âge verbal inférieur à 4 ans ( 5 de 5 ans, 4 de 6 ans et 1 de 7 ans), 5 égal à 4 ans (un de 5 ans, 4 de 7 ans) et 2 enfants de 7 ans présentent un âge verbal entre 4 et 6 ans selon les épreuves. Ces résultats confirment le trouble phonologique-syntaxique diagnostiqué par les professionnels. L'âge verbal des enfants typiques correspond à leur âge chronologique ou à un âge supérieur.

\section{Recueil des données}

Les dyades ont été invitées à participer à un jeu de devinettes lors duquel mère et enfant doivent, à tour de rôle, faire deviner à l'autre un item imagé sur une carte tirée dans une pioche. L'ensemble des éléments représentés isolément sur les cartes apparaissent également sur

3. À l'heure actuelle, les professionnels (logopédistes-orthophonistes, psychologues et neuropsychologues) francophones utilisent principalement cette classification pour établir le diagnostic des enfants présentant des troubles du langage. Elle correspond à celle de Rapin et Allen (1983). 
un plateau de jeu, représentant un jardin, que les participants ont sous les yeux. On demande aux participants de donner des indices permettant à leur interlocuteur de deviner l'item le plus rapidement possible. Les locuteurs sont tour à tour «indiçants» (ils donnent des indices) et «devinants» (ils cherchent à deviner l'item). L'indiçant doit élaborer, seul, une description ou une définition qui devrait permettre à son interlocuteur d'identifier l'item cible parmi un ensemble d'items possibles. Cela suppose d'une part la catégorisation de l'item et, d'autre part, la sélection des indices les plus pertinents dans le contexte du jeu. Cette activité langagière mobilise donc chez le locuteur à la fois des compétences linguistiques et des compétences pragmatiques.

\section{Analyse des données}

Les interactions ont été filmées et transcrites selon des conventions ${ }^{4}$ adaptées aux besoins de cette recherche. Dans une séquence de devinette, les interventions jouent des fonctions diverses, telles que proposer des indices, demander confirmation, acquiescer, proposer une solution, accepter ou refuser une proposition de solution, expliquer, piocher une carte, avancer les pions, comme l'illustre l'exemple 1 :

\section{(1) : Léo - 7;5 ans D - Item : Robinet}

Léo 18 - ((pioche une nouvelle carte $)) \mathrm{mm}::$ c'est un

Mèr 8 - ((s'adresse à l'observatrice $))$ c'est chacun son tour c'est ça?

Exp 6- oui c'est ça oui .

Mèr 9 - oui c'est ça .

Léo 19 - ça $=(($ mimant de la main droite l'action de tourner)) //voilà :: // $\{\mathrm{xxx}\}$

Mèr 10 - c'est un :: robinet.$=(($ pointe du doigt le dessin de l'objet en question sur le plateau de jeu))

Léo 20 - oui .hop $!=(($ avance son pion $))$ hop $=(($ jette sa carte sur le plateau de jeu devant sa mère))

Mèr 11 - $\quad((\text { pose la carte jouée sur la table }))^{\circ}$

L'analyse, qui ne concerne ici que les interventions correspondant à la formulation des indices (cf. (1), Léo 19, en gras), se déploie sur trois axes :

4. Les conventions de transcription n'ont pas fait l'objet d'une publication, mais sont rassemblées dans le rapport final adressé au FNRS en décembre 2008. Le lecteur intéressé peut prendre contact avec le $1^{\mathrm{er}}$ auteur. 
- les modalités des interventions de devinette selon que les indices sont exprimés uniquement de façon verbale, non verbale ou mixte; après avoir examiné les données globalement, nous nous intéressons à l'hétérogénéité intra-groupes et intra-dyades (styles des locuteurs et des dyades);

- l'analyse sémiologique des gestes qui porte sur le type de geste (déictique, figuratif, mimétique ou illustratif);

- le rôle du non-verbal par rapport au verbal qui se décline selon que le non-verbal entretient avec le discours une relation de complétion, de redondance ou de substitution.

La présentation détaillée des catégories d'analyse est donnée en introduction de l'exposé des résultats, pour chacun des axes. Les résultats sont présentés en alliant le quantitatif au qualitatif et en distinguant à chaque fois les populations $\mathrm{D}$ et $\mathrm{T}$. Le cas échéant, des analyses statistiques ont été réalisées (test de Mann-Whitney). La première série d'analyses a été conduite sur les interventions, unités fonctionnelles du dialogue. L'unité d'analyse des deuxième et troisième axes a été l'indice, composant des interventions de devinette (une intervention de devinette pouvant en effet comporter un - ex. 4, ci-dessous - ou plusieurs indices - ex. 2 ci-dessous).

\section{Modalités des interventions}

Chaque intervention des locuteurs a été caractérisée selon qu'elle est :

- seulement verbale :

(2) : Maé - 4;8 ans D - Item : tracteur

Mae 70 - euh i: c'est rouge et pis les roues i sont [plO].

- mixte (combinant verbal et non verbal) :

(3) : Dany - 6;8 ans D - Item : tracteur

Dan 98 - le soleil est derrière . $=(($ pointe vers le soleil $))$

- ou exclusivement non verbale ${ }^{5}$ :

(4) : Damien - 7;4 ans T - Item : oiseau

Dam 9 - ((mime l'oiseau qui vole $))$

5. Dans la mesure où nous n'analysons ici que les énoncés de devinette, l'appellation de non verbal désigne essentiellement les gestes. 


\section{Résultats globaux}

Le graphique 1 montre que les interventions de devinette (614 interventions pour les enfants, 606 pour les mères) sont formulées très majoritairement de façon uniquement verbale $(82,8 \%)$; une faible partie apparait sous une forme mixte $(14,4 \%)$; quelques-unes sont uniquement gestuelles $(2,8 \%)$. Ces premiers résultats attestent la nature essentiellement verbale de l'activité.

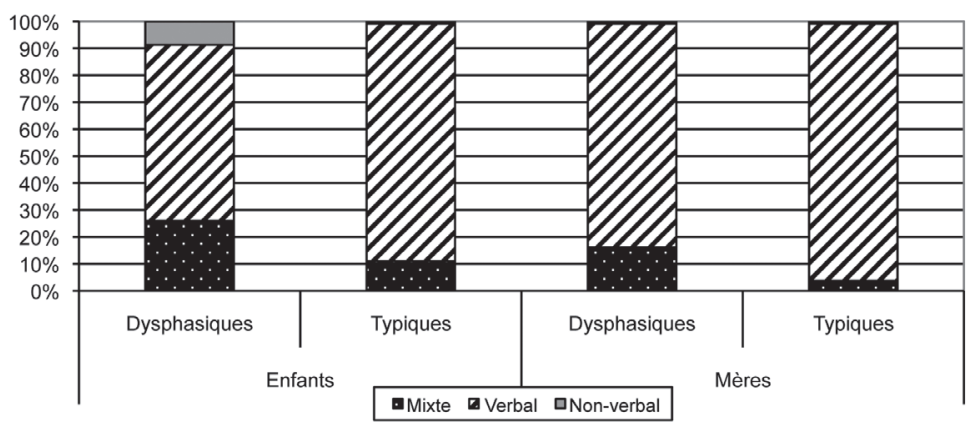

Graphique 1. - Distribution en pourcentages des modalités des interventions de devinette chez les enfants $D$, leurs pairs $T$ et leurs mères.

Même si tous les enfants produisent majoritairement des interventions verbales (minimum 65\%), les sujets D s'opposent aux sujets T par leur recours plus fréquent aux gestes, que ce soit de façon mixte (26,0\%; $\mathrm{T}: 11,0 \%$; différence significative : $\mathrm{U}=86,5 ; \mathrm{n} 1=18 ; \mathrm{n} 2=18$; $\mathrm{p}=, 016)$ ou purement non verbale $(8,7 \% ; \mathrm{T}: 0,41 \%$; différence significative : $\mathrm{U}=106,0 ; \mathrm{n} 1=18 ; \mathrm{n} 2=18 ; \mathrm{p}=, 015)$. Leurs interventions verbales sont par conséquent moins nombreuses $(65,3 \% ; \mathrm{T}: 88,6 \%$; différence significative : $\mathrm{U}=81,0 ; \mathrm{n} 1=18 ; \mathrm{n} 2=18 ; \mathrm{p}=, 010)$. Quant aux mères, leurs résultats présentent quelques analogies avec ceux des enfants. La tendance majoritaire à produire des interventions verbales se retrouve (D : 83,2\%; T : 95,9\%). Elle est toutefois un peu moins marquée chez les mères des enfants $\mathrm{D}$ qui font un usage plus fréquent des gestes, accompagnant essentiellement le verbal dans des interventions mixtes $(16,2 \%$; différence significative : $\mathrm{U}=97,5 ; \mathrm{n} 1=18 ; \mathrm{n} 2=18$; $\mathrm{p}=, 031)$ que les mères des enfants $\mathrm{T}(3,76 \%)$. Les mères de ces derniers ont recours au gestuel de façon résiduelle. 
Ainsi, les dyades avec des enfants $\mathrm{D}$, bien que formulant des énoncés de devinette de façon préférentiellement verbale, intègrent davantage que les dyades avec un enfant $\mathrm{T}$ le gestuel comme un moyen de donner des indices à leur interlocuteur. Si cette observation est plus claire pour les enfants, cela est malgré tout également le cas pour leurs mères qui adoptent aussi quelque peu ce comportement.

\section{Styles des dyades}

L'observation des données nous amène également à constater des différences inter-dyades, certaines recourant à l'évidence davantage aux gestes que d'autres. Se pose alors la question de l'homogénéité des dyades. Autrement dit, mère et enfant ont-ils tendance à adopter des comportements analogues? Si oui, peut-on dégager un style pour chaque dyade dans l'utilisation des gestes?

Afin de répondre à ces questions, nous avons procédé à deux analyses complémentaires. Nous avons tout d'abord caractérisé le degré de gestualité des sujets (mères et enfants séparément) en les classant en cinq catégories de sujets, par ordre croissant en fonction de l'importance de la mobilisation des gestes utilisés dans les interventions de devinette, en termes de pourcentage des interventions comprenant des gestes :

- les non gestuels (NG) : à $0-1 \%$ des interventions ;

- les petits gestuels (PG) : entre $1 \%$ et $6 \%$;

- les moyens gestuels (MG) : de $7 \%$ à $20 \%$;

- les grands gestuels (GG) : de $21 \%$ à $35 \%$;

- les très grands gestuels (TG) : dès $36 \%$.

La catégorie MG est déterminée comme celle regroupant les sujets se situant autour de la moyenne. Deux autres catégories en dessous et en dessus du groupe moyen ont été ensuite établies. Dans la mesure où la distribution des pourcentages de gestes présente une grande concentration de sujets dans les valeurs situées entre la moyenne et 0 et une plus grande dispersion dans les valeurs supérieures à la moyenne, les intervalles définissant les groupes ne sont pas symétriques. Compte tenu du faible nombre de sujets par catégorie et de l'absence de symétrie des groupes ainsi définis, la présentation des résultats est réalisée uniquement à des fins descriptives. 


\begin{tabular}{|c|c|c|c|c|c|c|c|}
\hline \multicolumn{2}{|c|}{ Sujets } & NG & PG & MG & GG & $\mathrm{TG}$ & Total \\
\hline \multirow{3}{*}{ Enfants } & $\mathrm{D}$ & 2 & - & 4 & 5 & 7 & 18 \\
\hline & $\mathrm{T}$ & 6 & 3 & 5 & 3 & 1 & 18 \\
\hline & Total & 8 & 3 & 9 & 8 & 8 & 36 \\
\hline \multirow{3}{*}{ Mères } & $\mathrm{D}$ & 6 & 2 & 5 & 3 & 2 & 18 \\
\hline & $\mathrm{T}$ & 10 & 4 & 4 & - & - & 18 \\
\hline & Total & 16 & 6 & 9 & 3 & 2 & 36 \\
\hline \multicolumn{2}{|c|}{ Total } & 24 & 9 & 18 & 11 & 10 & 72 \\
\hline
\end{tabular}

Tableau 1. - Distribution des sujets selon le taux de gestes dans leurs interventions de devinette (chiffres absolus).

Le tableau 1 met en évidence que les enfants, pris individuellement, tendent à avoir plus souvent recours à des gestes que leurs mères, et plus particulièrement les enfants $\mathrm{D}$, puisqu'ils sont presque tous minimalement des sujets «moyens gestuels». Les 5 mères appartenant aux catégories GG et TG sont toutes des mères d'enfants D.

Cependant nous avons à faire autant à des styles individuels qu'à des styles de dyades. En effet, mère et enfant peuvent montrer des conduites similaires ou au contraire diverger dans leur recours aux gestes. Nous avons évalué le degré d'homogénéité des dyades en prenant en considération uniquement la différence de pourcentages de gestes entre la mère et l'enfant : dans les dyades homogènes, mère et enfant ont moins de $10 \%$ de différence; les dyades hétérogènes se caractérisent par une différence de plus de $10 \%$.

20 dyades sont homogènes et 16 hétérogènes, les dyades avec un enfant $\mathrm{T}$ étant plus souvent homogènes (12 dyades sur 18) que celles avec un enfant $\mathrm{D}$ ( 8 dyades sur 18$)$. Quant aux dyades hétérogènes, à l'exception d'une dyade avec une enfant $\mathrm{D}$, les enfants produisent plus de gestes que leur mère.

\section{Analyse sémiologique des gestes}

La question de la catégorisation sémiologique et/ou fonctionnelle de la kinésie communicative reste un problème en soi, en particulier parce que, comme l'a bien montré Colletta (2004), les critères sémiologiques et fonctionnels sont souvent amalgamés dans les catégorisations proposées dans la littérature (Cosnier et al., 1982; Kendon, 1988). Or la fonction des mêmes gestes varie selon qu'ils sont produits seuls ou associés 
au discours. Dans la mesure où nous nous centrons sur la façon dont le gestuel est mobilisé pour l'élaboration et la transmission des indices, nous avons pris le parti de ne travailler que sur les gestes référentiels. Les autres modes de participation de l'enfant à l'échange sont donc volontairement exclus. À partir des propositions de Colletta (2004), nous relevons dans le corpus quatre types de gestes associés à la production d'indices :

- les déictiques (DEI) qui désignent le référent dans le contexte situationnel dont l'exemple 3 est caractéristique. Ils correspondent à divers pointages (du doigt, de la main...) et autres formes de balayage ou de délimitation de l'espace :

(5) : Natacha - 6;1 ans D - Item : ballon

Nat 7 - c'est / quelque [soz] que c'est / [a] bas ((montre le bas du plateau $))$ et c'est // pas ici . = $(($ sépare verticalement de la main le plateau)) // c'est pas ici . $=(($ sépare à nouveau verticalement de la main le plateau)) //

- les illustratifs (ILL) (pictomimiques chez Cosnier) qui représentent la forme ou d'autres qualités du référent :

(6) : Émilie - 7;10 ans D - Item : ballon

Emi 73 - et pis c'est rond . = ((fait un geste circulaire de la main))

- les mimétiques (MIM) (kinémimiques chez Cosnier) qui miment une action, du référent (voir exemple 4) ou l'impliquant (voir exemple 1);

- les figuratifs (FIG) qui «représentent par métaphore ou métonymie un référent abstrait» (Colletta, 2004, p. 169) :

(7) : Mère de Dany - 6;8 ans D - Item : oiseau

Mèr 71 - quel(e)qu(e) chose qui va:: très loin : à = ((fait un geste circulaire au-dessus de sa tête))

- certains gestes restent ininterprétables (ININT).

Le graphique 2 met en évidence que les gestes déictiques dominent pour tous les groupes et à tous les âges. Chez certains enfants $(6 \mathrm{D}$ et $5 \mathrm{~T}$ ) et certaines mères ( $3 \mathrm{D}$ et $3 \mathrm{~T}$ ), il s'agit même du seul type de geste mobilisé. Les gestes mimétiques constituent le deuxième type de geste mobilisé, en proportion moindre, sauf pour les enfants $\mathrm{T}$ chez qui ils occupent une place comparable à celle des déictiques. Les autres catégories ne comprennent que très peu d'occurrences (au maximum 10). 


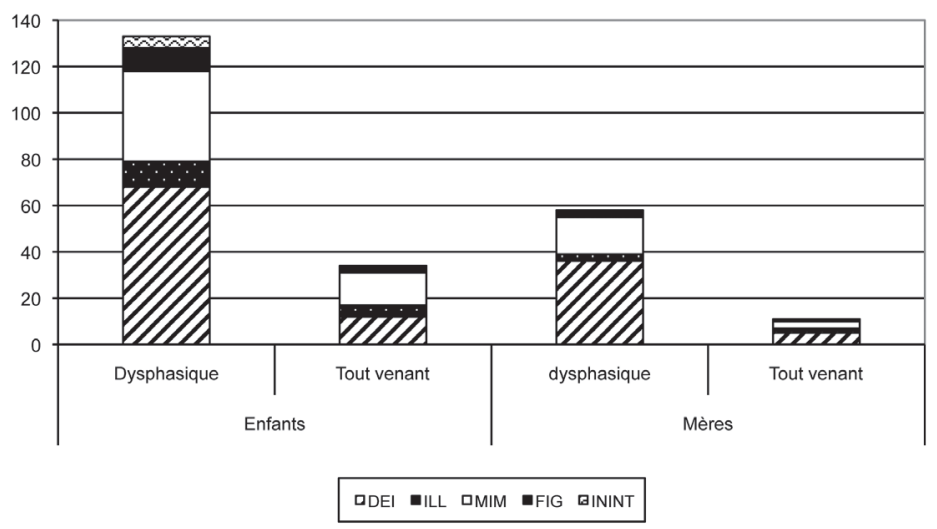

Graphique 2. - Distribution des types de gestes contribuant à la formulation des indices (chiffres absolus) chez les enfants $D$, leurs pairs $T$ et leurs mères.

\section{Les gestes déictiques}

Ces gestes véhiculent le plus souvent (96 cas) un acte de localisation. Celui-ci peut être isolé (comme dans (3) et (5)) ou se combiner avec d'autres stratégies de codage (par exemple, une description de l'item ou une catégorisation générique).

(8) : Mère de Julie1 - 6;3 ans D - Item : drapeau

Mer 176 - ça vole assez vite dans le ciel c'est : / bleu rose un peu d(e) gris . / c'est là [...]. $=(($ montre le haut du plateau))

Cette stratégie de localisation est mobilisée de façon dominante par les enfants D (53 gestes déictiques sur 69).

Dans d'autres cas ( 8 cas), le geste pointe un autre item posé comme terme de comparaison ou d'exclusion :

(9) : Dylan - 5;6 ans D - Item : chien

Dyl $166-$ pas ça.$=(($ pointe un animal sur le plateau de jeu $))$

Enfin, les cas où le geste déictique vient en appui d'un autre type d'indice, comme dans (10), sont relativement peu fréquents (14 cas) :

(10) : Léo - 7;5 ans D - Item : ours

Leo 62 - ça. // marron . = ((fait le tour de son œil avec son index)) 
Chez les mères des enfants $\mathrm{D}$, la localisation de l'item correspond également à la grande majorité de leurs gestes déictiques (23 sur 36).

\section{Les gestes référentiels non déictiques}

Contrairement aux précédents, les gestes référentiels non déictiques contribuent à la représentation de l'item à deviner. Cette représentation correspond à diverses stratégies de codage, telles que l'évocation de traits définitoires (catégories, actions, savoirs encyclopédiques liés à l'item) ou des caractéristiques particulières de l'item représenté.

Les gestes illustratifs représentent ainsi des traits typiques de l'item :

(11) : Thierry - 7;5 ans D - Item : coq

Thi 64 - ((mime : accroupi, mains unies devant la bouche et marche dans cette position autour de la table))

Cette stratégie est relativement fréquente chez les enfants D qui produisent des gestes non déictiques (8 enfants sur 10) et moins fréquente chez les enfants $\mathrm{T}$ ( 3 sur 7 ). Elle est plus rare chez les mères ( 2 mères d'enfants $\mathrm{D}$ et 2 mères d'enfant $\mathrm{T}$ ).

À l'opposé, les gestes mimétiques miment les actions typiques des items (exemple 12) ou impliqués par les items (exemple 1) :

(12) : Léo - 7;5 ans D - Item : ours

Leo 48 - ((mime l'action de grimper les doigts crochus, 3 sec.)) et c'est marron . voilà! //

Cette stratégie est présente chez 5 des 7 enfants $\mathrm{T}$ et moins fréquente chez les enfants D. En effet, un seul parmi ces derniers, Arthur, concentre à lui seul 27 des 39 occurrences des gestes mimétiques; les 12 occurrences restantes se répartissent entre 4 enfants D.

Même si on retrouve cette stratégie chez 6 des 10 mères d'enfants $D$ produisant des gestes non déictiques, la mère d'Arthur, comme son fils, produit à elle seule 9 des 16 gestes mimétiques, type qui constitue d'ailleurs pour elle sa stratégie préférentielle. On constate donc également sur ce plan une importante hétérogénéité des dyades.

\section{Rôle des gestes en relation au discours}

Les énoncés d'indices ont été caractérisés selon le rapport que le gestuel entretient avec le verbal. La relation des gestes au discours a été diversement caractérisée dans la littérature. Nous proposons, en nous inspirant de Colletta (2004), trois types de rôle : 
- Substitution : le gestuel se substitue au verbal (SUB). L'intervention gestuelle est le seul vecteur de signification, dans une intervention non verbale (dans [4] et [11]) ou mixte (12);

- Complétion : le gestuel apporte, en complément, une signification au message verbal, il lui est complémentaire (COM), comme dans (1) et (5);

- Redondance : le gestuel apporte les mêmes éléments de signification que le verbal (RED), comme dans (3) et (6).

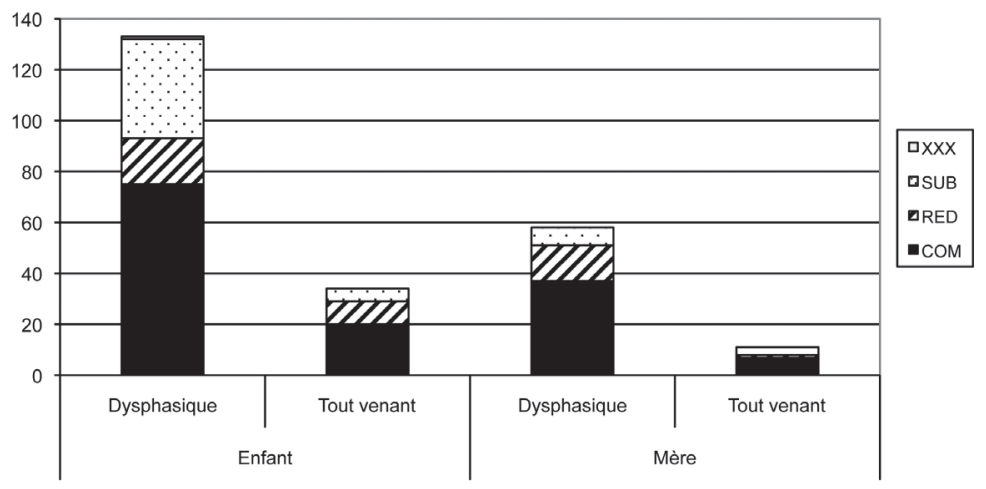

Graphique 3. - Distribution des gestes selon leur relation au discours (nombres absolus).

La tendance majoritaire de l'ensemble des dyades (cf. graph. 3) consiste en une utilisation complémentaire du gestuel par rapport au verbal : en effet sur les 236 gestes répertoriés, 139 jouent un rôle complémentaire $(\mathrm{COM})$. Ces gestes viennent fréquemment ajouter une information au message verbal, et deviennent souvent indispensables à la compréhension de l'intervention, surtout dans le cas des gestes accompagnant les déictiques verbaux (comme dans [1], [5], [8] et [9]). Les enfants (populations confondues) ont aussi tendance à produire plus de gestes se substituant au verbal que leurs mères ( $\mathrm{SUB}: \mathrm{U}=517,0$; $\mathrm{n} 1=18 ; \mathrm{n} 2=18 ; \mathrm{p}=, 047)$ ou lui étant redondant (RED : $\mathrm{U}=469,5$; $\mathrm{n} 1=18 ; \mathrm{n} 2=18 ; \mathrm{p}=, 028)$. À nouveau, des différences s'observent entre les deux groupes d'enfants, les D produisant davantage que les $\mathrm{T}$ de gestes en complétion $(\mathrm{U}=85,0 ; \mathrm{n} 1=18 ; \mathrm{n} 2=18 ; \mathrm{p}=, 013)$. En même temps, aucune différence statistiquement significative entre les groupes n'apparait, ni pour la redondance ni pour la substitution. Cette absence de significativité s'explique par le fait que les chiffres 
correspondent essentiellement aux données de deux enfants D (Arthur, 22 occurrences et dans une moindre mesure Thierry, 8 occurrences). Par ailleurs, d'un point de vue qualitatif, les enfants $T$ utilisent des gestes en substitution au sein d'interventions de devinette complexe, en combinaison avec des indices verbaux, ce que ne font pas les enfants D.

En ce qui concerne les mères, on observe uniquement que les gestes des mères des enfants $\mathrm{D}$ sont plus souvent que ceux des mères des enfants $\mathrm{T}$ en relation de redondance avec le verbal $(\mathrm{U}=105,5 ; \mathrm{n} 1=18$; $\mathrm{n} 2=18 ; \mathrm{p}=, 023)$.

\section{Discussion et conclusion}

Nous nous sommes centrés dans cette étude sur la contribution des gestes à la formulation des indices dans une activité de devinette, c'està-dire à la façon dont les gestes peuvent être mobilisés dans une tâche de catégorisation, description et, plus généralement, dans le versant idéationnel de la communication. Sur le plan verbal, ce type de tâche s'est révélé plus difficile pour les enfants dysphasiques que pour les enfants typiques (Salazar Orvig et al., 2007), leurs indices s'avérant souvent insuffisants, voire non congruents. Ils produisent ainsi des descriptions moins variées au moyen de stratégies également moins adaptées à l'interlocuteur et à l'item particulier à faire deviner.

Les résultats de l'étude des gestes mettent en évidence une différence nette de fonctionnement des dyades $\mathrm{D}$ par rapport aux dyades $\mathrm{T}$. En premier lieu, même si globalement la devinette se profile comme une activité essentiellement verbale, il apparait que les enfants mobilisent plus souvent les gestes pour la production d'indices que leurs mères et que les dyades $\mathrm{D}$ les intègrent davantage comme un moyen de construire des indices pour leur interlocuteur.

En ce qui concerne les enfants, ce constat rejoint les travaux dans la littérature qui montrent que les enfants $\mathrm{D}$ recourent davantage au nonverbal que leurs pairs T (Bishop et al., 1998; Mermoud et de Weck, 1992; Thal et Tobias, 1992). Cependant, comme cela a été signalé en introduction, on relève une divergence entre les recherches précitées et celles qui, au contraire, concluent à une absence de différence sur le plan quantitatif entre les populations (Blake et al., 2008; Botting et al., 2010). Cette divergence pourrait s'expliquer par la spécificité de la situation de devinette. Comme Blake et al. (2008) l'ont noté, la production de gestes est fortement dépendante du genre discursif et de l'activité en cours. 
En outre, la comparaison avec les enfants $\mathrm{T}$ a mis en évidence la spécificité de la communication des enfants $\mathrm{D}$, puisque leurs comportements ne correspondent ni à ceux de leurs pairs d'âge ni à ceux d'enfants plus jeunes.

En effet, si on s'attache aux productions en fonction de l'âge, ce que faute de place nous n'avons pu décrire dans cet article, on peut noter que le recours au non-verbal augmente avec l'âge et qu'il est bien plus important chez les enfants D les plus âgés (groupe des 7 ans). Ainsi, comme certains auteurs l'on clairement dit (Blake et al., 2008), il n'y aurait pas de rapport simple entre niveau linguistique et recours aux gestes. Ce recours pourrait, par exemple, ne pas correspondre à un comportement primitif mais à l'effet de l'expérience communicative chez certains enfants et au développement de conduites leur permettant de compenser par la modalité gestuelle les difficultés verbales rencontrées : lorsque leurs moyens langagiers ne leur permettent pas de donner des indices de façon satisfaisante, ils parviennent à compléter leurs interventions par un geste, voire à remplacer une formulation verbale par un geste significatif, ce qui leur permet d'assumer leur rôle dans l'activité langagière. Ce constat rejoint également certaines données de la littérature (Evans et al., 2001; Blake et al., 2008, par exemple).

En même temps, il apparait que le recours au non-verbal n'est pas homogène chez tous les sujets. Dans les deux populations, certains enfants ne produisent pas (ou très peu) de gestes dans la formulation des indices, alors que d'autres en présentent un taux relativement important. Cette hétérogénéité de la population suggère que l'on a aussi affaire à des profils individuels qui évoluent avec l'âge, et que, dans le cas de la dysphasie, ce recours au gestuel vient moduler les troubles dysphasiques, permettant à certains enfants dysphasiques d'être plus efficaces que d'autres dans l'interaction.

L'analyse de la dimension sémiologique des gestes a mis en évidence l'importance des gestes déictiques chez les enfants $\mathrm{D}$, gestes qui correspondent à une stratégie de localisation de l'item. Si cette stratégie est présente chez la plupart des enfants, elle semble constituer la conduite prédominante pour certains des enfants (D mais aussi parmi les $\mathrm{T}$ les plus jeunes). Même si ces pointages ne correspondent pas nécessairement à la désignation directe de l'item à deviner, cette stratégie correspond à une façon d'accomplir la tâche fortement ancrée dans la situation matérielle de production : se basant sur un savoir préalablement posé par la consigne (les items sont représentés sur le plateau de jeu), le locuteur ne procède pas à une catégorisation de l'item, ni à 
la sélection des traits pertinents qui pourraient permettre à son interlocuteur de trouver la solution rapidement. Restant rivés au plateau, ces enfants semblent avoir de la difficulté à se détacher du hic et nunc de l'activité concrète et à mener à bien un travail d'abstraction. Une difficulté similaire a été mise en évidence chez les enfants D dans d'autres situations discursives (Uzé et Stonehouse, 1996).

D'un autre côté, les deux enfants D qui mobilisent le plus de gestes non déictiques présentent également une très forte proportion de gestes. Ils produisent surtout des gestes mimétiques ou illustratifs, proposant donc à leur interlocuteur une «incarnation» de l'item à deviner. En outre, ces gestes se substituent le plus souvent totalement au discours. Ils sont les seuls indices fournis à leur interlocuteur. L'étude d'Evans et al. (2001) a mis en évidence un phénomène semblable dans une tâche d'explication : les enfants D exprimaient par des gestes des informations non contenues dans leur discours. À l'opposé, les enfants $\mathrm{T}$ qui ont recours à ce type de geste le font en association avec des indices verbaux dans une relation de complétion. Ainsi se dessinent des profils différents d'enfants dysphasiques et typiques.

La gestualité co-verbale (Iverson et Goldin-Meadow, 2001; McNeill, 2002) n'a pas uniquement une fonction interactive, elle est également constitutive du travail d'élaboration langagière. De ce point de vue la devinette, par le travail d'évocation de concepts qu'elle suppose, constitue en soi une situation favorisant la production de gestes. Cependant, de l'analyse de nos données, il apparait que les difficultés constatées chez les enfants D pour élaborer les devinettes se reflètent dans des stratégies spécifiques sur le plan gestuel: en effet, le geste ne semble pas constituer un support dans la conduite idéationnelle mais un outil sémiotique leur permettant malgré tout d'endosser leur rôle d'indiçant et ainsi de réaliser l'activité langagière proposée.

En ce qui concerne les mères, il apparait que même si elles produisent moins de gestes que leurs enfants (globalement et prises individuellement), ce sont les mères des enfants $\mathrm{D}$ qui en utilisent le plus. De même, comme leurs enfants, elles ont très souvent recours à des gestes déictiques. Si ces gestes s'inscrivent dans une relation de complétion avec le discours, c'est aussi chez ces mères que l'on constate proportionnellement plus de gestes redondants avec le discours. Ainsi, l'analyse des gestes nous permet de toucher du doigt une facette de l'adaptation des mères à leurs enfants, puisque l'on observe une différence selon que leur enfant présente ou non des troubles du développement du langage. On assiste chez les mères à deux types de conduite : d'une part, une 
certaine empathie avec leur enfant, empathie qui s'exprime par l'importance des dyades homogènes et par le recours à des stratégies sémiologiques similaires, en particulier la localisation ou le mime; d'autre part, contrairement à leurs enfants, les mères des enfants $\mathrm{D}$ vont parfois soutenir leurs interventions verbales avec un geste qui a la même signification que le verbal, afin de s'assurer vraisemblablement que leur enfant comprend bien ce qu'elles disent et contribuer ainsi à la réussite de la tâche.

Ce comportement gestuel et son effet sur la compréhension ont été mis en évidence dans certaines études (Ellis, Weismer et Hesketh, 1993) sur tous les enfants, mais plus particulièrement chez les enfants D. Le profil des mères étudiées ici semble confirmer une adaptation au niveau langagier de leur enfant comme les mères des enfants plus jeunes (Iverson et al., 1999) ou celles des enfants souffrant du syndrome de Down (Iverson et al., 2006).

En même temps, et conformément aux données de la littérature, il apparait aussi que les mères ne constituent pas des groupes homogènes. Des styles individuels contrastés doivent être pris en compte. La confrontation de ces conduites à celles développées dans d'autres situations de communication permettra de faire la part de l'interaction entre stratégies induites par la tâche et adaptation au niveau langagier de l'enfant.

\section{RÉFÉRENCES BIBLIOGRAPHIQUES}

Bishop D. V. M. (2000) : «Pragmatic language impairment: A correlate of SLI, a distinct subgroup, or part of the autistic continuum?», dans D. V. M. Bishop et L. B. Leonard (éds), Speech and language impairments in children: causes, characteristics, intervention and outcome, Hove, Psychology Press, p. 99-113.

Bishop D. V. M., Chan J., Adams C., Hartley J. et Weir F. (2000) : «Conversational responsiveness in specific language impairment: Evidence of disproportionate pragmatic difficulties in a subset of children», Development and Psychopathology, vol. 12, n ${ }^{\circ}$ 2, p. 177-190.

Bishop D. V. M., Chan J., Hartley J. et Weir F. (1998) : «When a nod is as good as a word : Form-function relationships between questions and their responses», Applied Psycholinguistics, vol. 19, n 3, p. 415-432.

Blake J., Myszczyszyn D., Jokel A. et Bebiroglu N. (2008) : «Gestures accompanying speech in specifically language-impaired children and their timing with speech», First Language, vol. 28, n 2, p. 237-253. 
Botting N., Riches N., Gaynor M. et Morgan G. (2010) : «Gesture production and comprehension in children with specific language impairment», British Journal of Developmental Psychology, vol. 28, $\mathrm{n}^{\circ} 1$, p. 51-69.

Capone N. C. et McGregor K. K. (2004) : «Gesture Development: A Review for Clinical and Research Practices», Journal of Speech, Language, and Hearing Research, vol. 47, $\mathrm{n}^{\circ}$ 1, p. 173-186.

Chevrie-Muller C. et Narbonna J. (éds) : (2000) : Le langage de l'enfant. Aspects normaux et pathologiques, Paris, Masson.

Chevrie-Muller C. et Plaza M. (2001) : Nouvelles épreuves pour l'Examen $d u$ Langage ( $N$-EEL), Paris, ECPA.

Colletta J.-M. (2004) : Le développement de la parole chez l'enfant âgé de 6 à 11 ans, Sprimont, Mardaga.

Conti-RAMSDEn G. (1994) : «Language interaction with atypical language learners », dans C. Gallaway et B. Richards (éds), Input and Interaction in Language Acquisition, Cambridge, CUP, p. 183-196.

Conti-Ramsden G., Hutcheson G. D. et Grove J. (1995) : «Contingency and breakdown: Children with SLI and their conversations with mothers and fathers», Journal of Speech and Hearing Research, vol. 38, p. 12901302.

COSNIER J. (1977) : «Communication non verbale et langage», Psychologie Médicale, vol. 9, $\mathrm{n}^{\circ}$ 11, p. 2033-2049.

Cosnier J., Coulon J., Berrendonner A. et Orecchioni C. (éds) (1982) : Les voies du langage, Paris, Dunod.

DAHAN G. et CosNier J. (1977) : «Sémiologie des quasi-linguistiques français», Psychologie Médicale, vol. 9, n 11, p. 2053-2072.

Eluis Weismer S. et Hesketh L. J. (1993) : «The influence of prosodic and gestural cues on novel word acquistion by children with specific language impairment », Journal of Speech and Hearing Research, vol. 36, $\mathrm{n}^{\circ}$ 5, p. 1013-1025.

Evans J. A., Alibali M. W. et McNeill N. M. (2001) : «Divergence of verbal expression and embodied knowledge: evidence from speech and gesture in children with specific language impairment», Language and Cognitive processes, vol. 16, $\mathrm{n}^{\circ}$ 2-3, p. 309-331.

GÉRARD C. L. (1991) : L'enfant dysphasique, Paris, Éditions universitaires.

HuPET M. (1996) : «Troubles de la compétence pragmatique : troubles spécifiques ou dérivés?», dans G. de Weck (éd.), Troubles du développement du langage. Perspectives pragmatiques et discursives, LausanneParis, Delachaux et Niestlé, p. 89-112.

Iverson J. M., Capirci O., Longobardi E. et Caselli M. C. (1999) : «Gesturing in mother-child interactions», Cognitive Development, vol. 14, $\mathrm{n}^{\circ} 1$, p. $57-75$. 
Iverson J. M. et Goldin-MeAdow S. (2001) : «The resilience of gesture in talk: Gesture in blind speakers and listeners », Developmental Science, vol. 4, no 4, p. 416-422.

Iverson J. M., Longobardi E., Spampinato K. et Caselli M. C. (2006) : «Gesture and speech in maternal input to children with Down's syndrome », International Journal of Language \& Communication Disorders, vol. 41, n 3, p. 235-251.

Kendon A. (1988) : «How gestures can become like words», dans F. Potyatos (éd.), Cross-cultural perspectives in nonverbal communication, Toronto, Hogrefer, p. 131-141.

McNeILl D. (2002) : «Gesture and language dialectic», Acta linguistica Hafniensia, vol. 34, p. 7-37.

Mermoud L. et DE WeCK G. (1992) : «Étude clinique de la mimogestualité d'une enfant dysphasique», Glossa, n 32, p. 44-49.

Panagos J. M., Bobkoff Katz K., Kovarsky D. et Prelock P. A. (1988) : «The nonverbal component of clinical lessons », Child Language Teaching and Therapy, vol. 4, no 3, p. 278-296.

Rapin I. et Allen D. A. (1983) : «Developmental language disorders », dans U. Kirk (éd.), Neuropsychology of language, reading and spelling, New York, Academic Press, p. 155-184.

Salazar Orvig A. et DE Weck G. (2008) : «Profils dialogiques de dyades mère-enfant avec et sans troubles du langage», TRANEL, $\mathrm{n}^{\circ}$ 49, p. 45-67.

Salazar Orvig A., de Weck G., Basselier M. et Henry A. (2007) : «Dialogue entre enfants dysphasiques et leur mère : analyse des processus d'ajustement », Rééducation Orthophonique, n 230, p. 25-52.

Thal D. J. et Tobias S. (1992) : «Communicative gestures in children with delayed onset of oral expressive vocabulary », Journal of Speech and Hearing Research, vol. 35, nº 6, p. 1281-1289.

Uzé J. et Stonehouse S. (1996) : «L'activité de récit chez l'enfant dysphasique», A.N.A.E., n 39-40, p. 127-131.

Weck (DE) G. et Rosat M. (2003) : Troubles dysphasiques. Comment raconter, relater, faire agir à l'âge pré-scolaire, Paris, Masson.

WeCk (DE) G. et SAlazAR ORvig A. (2010) : «Les interactions mère-enfant dysphasique : qu'y a-t-il encore à comprendre?», Langage \& Pratiques, $\mathrm{n}^{\circ} 46$. 